\title{
El traductor y el Comercio Internacional
}

\author{
María Ángeles Orts Llopis \\ Universidad de Murcia \\ mageorts@um.es
}

Resumen: La internacionalización de la economía ha hecho necesaria la implementación de una red de estrategias comunicativas de la empresa a nivel global, en las que se requieren especialistas multilingües y multiculturales. Exportador e importador han de enfrentarse a los distintos agentes y actividades que intervienen en su salida al exterior en una lengua o lenguas que les son a menudo desconocidas. Por ello, el de la internacionalización es un campo que está especialmente destinado al traductor formado en esta área, que se torna aquí en agente comunicativo, intérprete y mediador cultural. El presente trabajo se centra en la descripción del contexto donde este profesional desarrolla su labor y de las actividades que está capacitado para desempeñar.

Palabras clave: Comercio Internacional; traductor en el ámbito del Comercio Internacional; internacionalización; mediación cultural; estrategia comunicativa.

\section{The translator in foreign trade}

Abstract: The internationalization of economy requires the implementation of a network of corporate communication strategies at global level, in which multilingual and multicultural specialists are required. Exporter and importer have to deal with the different parties and activities involved in their overseas activities in a language or languages that are often unknown to them. Therefore, internationalization is a field that is custom-made for translators trained in this area, where they turn into communication agents, interpreters and cultural mediators. This paper focuses upon the description of the context in which this professional develops her/his work, and upon the activities she/he is qualified to perform.

Keywords: foreign trade; international commercial translator; internationalization; communication strategy. 



\section{Introducción: la empresa española y las estrategias lingüísticas de internacionalización}

El presente trabajo está enfocado hacia la descripción de la actividad del traductor en el ámbito del Comercio Internacional y la justificación de su figura como fundamental en el desarrollo del proceso de internacionalización de las empresas. En primer lugar, es necesaria una contextualización de lo que representa el Comercio Internacional en España, procediendo a la descripción de las necesidades comunicativas de una empresa española que se internacionaliza y, posteriormente, detallar cuáles han de ser la formación y funciones que puede ejecutar el traductor en el ámbito del Comercio Internacional en estas. El tercer punto será tratado extensamente en las siguientes secciones de este trabajo.

Para entender el papel primordial que ostenta la internacionalización en el desarrollo óptimo de un país, hemos de resaltar que vivimos en una era de globalización, principalmente en el área de la economía y el comercio. El mundo todavía sufre las consecuencias de la llamada Gran Recesión de 2009, una crisis económica en parte debida a la globalización, que hace que las enfermedades financieras como la crisis de la Bolsa China de diciembre de 2015 se hayan transmitido en 2016 a Wall Street y de ahí al resto del mundo, como si de una letal pandemia se tratara.

Pero pese a esta prolongada crisis -y a las alarmantes cifras del Instituto Nacional de Estadística, que anuncian que la tasa de paro en España se sitúa en el 22,37\% , una de las más altas de la Unión Europea ${ }^{1}-$, el informe llamado Invest in Spain auspiciado por el Instituto de Comercio Exterior (ICEX) afirma que en los últimos años las exportaciones e importaciones españolas han crecido rápidamente, lo que convierte a España en uno de los países más internacionalizados del mundo, superando en marzo del 2015 los veintitrés mil millones de euros. Además, de acuerdo con los datos del Ministerio de Economía transmitidos por El País, la mejora de las ventas exteriores y el desplome del crudo redujeron el déficit comercial español un $1,1 \%$ en los nueve primeros meses del año 2015, hasta los 18.642 millones de euros. En concreto, las

\footnotetext{
${ }^{1}$ Instituto Nacional de Estadística: http://www.ine.es/daco/daco42/daco4211/epa0215. pdf.
} 
exportaciones repuntaron un 4,4\% entre enero y septiembre, hasta los 186.261 millones, mientras que las importaciones subieron un 3,9\%, hasta los 204.903 millones.



Gráfico 1: Evolución de las exportaciones e importaciones españolas en 2015.

El informe del ICEX mencionado anteriormente afirma, además, que los principales socios comerciales de España son los países de la UE27, seguidos de Asia y África, desbancando a América Latina y América del Norte en el puesto que tradicionalmente venían ocupando estos territorios como principales socios comerciales de España fuera de la UE. Como consecuencia, la promoción de las exportaciones y la internacionalización de las empresas pueden generar miles de nuevos puestos de trabajo en los próximos años, de forma que la apuesta por el sector exterior en España se considera, ahora más que nunca, uno de los mejores soportes para afrontar la crisis y generar empleo estable. Concretamente, el que haya una mayor profesionalización de los agentes que actúan en este sector beneficia enormemente al traductor formado en Comercio Internacional, proporcionándole ventajosas salidas laborales. El fomento de la exportación y el apoyo a la salida hacia otros mercados de las empresas españolas son una gran oportunidad para un individuo con capacidad comunicativa multilingüe y una sensibilidad desarrollada para entender las diferencias culturales de otros países. 
Por otro lado, una empresa que se internacionaliza es aquella que tiene la capacidad, los medios y la gestión apropiados para distribuir un producto comercial o servicio a escala global a un precio competitivo. Según la Guía básica del comercio internacional publicada por la Cámara de Comercio Internacional (2012), la compraventa de mercancías en el contexto global es esencial para una economía salubre, y por ello la mayoría de los gobiernos facilitan ayuda a las empresas que a ello se dedican. No hace falta ser una multinacional para internacionalizarse en España: las Cámaras de Comercio prestan ayuda en lo que se refiere a la formación e información de las empresas en sus procesos de internacionalización. De hecho, la búsqueda de nuevos mercados se presenta como una opción casi indispensable para la supervivencia de la PYME española, en un mundo en el que exportar ya no es una moda, sino casi una obligación para las empresas que quieran sobrevivir a la fiera competitividad del mercado (Solano Sánchez, 2014: 5). El negocio puramente local a la antigua usanza agoniza, si no ha muerto, y el empresario o emprendedor tiene que saber que no solo se enfrenta a barreras logísticas, jurídicas y financieras, sino también a otras del ámbito lingüístico y cultural.

Sin embargo, como también apunta la Cámara de Comercio Internacional en su Guía (2012: 12), exportar e importar conlleva riesgos, y abordar de manera profesional las operaciones de Comercio Internacional es el camino más seguro para gestionarlos. Pero, a su vez, esta profesionalización lleva inherentes factores de cariz comunicativo, en sus vertientes lingüística y cultural. Según el informe ELAN (Effects on the European Economy of Shortage of Foreign Language Skills in Enterprise) realizado por la Comisión Europea en 2006, casi 200 empresas europeas perdieron posibles contratos por no saber idiomas, 37 valoraron esta pérdida de negocio en una cifra de entre 8 millones y 13,5 millones de euros. Otras 54 empresas perdieron contratos por valor de entre 16,5 millones y 25,3 millones de euros, y 10 habían perdido contratos por valor de 1 millón de euros. De entre las razones detectadas por dichas empresas para esta pérdida de negocio se encontraba la incapacidad de su personal para hablar idiomas -lo que hizo imposible seguir las solicitudes de información o los presupuestos o atender correctamente llamadas del extranjero-, los errores de traducción o interpretación y la falta de afinidad cultural. En concreto, y tal y como apunta Aguayo Arrabal, el 'hermetismo', o el rechazo de las PYMES 
españolas para salir al exterior se debe a las dificultades que presenta el entablar relaciones comerciales en una lengua extranjera para sus proyectos internacionales, o la imposibilidad de sostener "las futuras relaciones que deban mantener con el exterior debido a su interés de expandir su mercado fuera de nuestras fronteras" (Aguayo Arrabal, 2013: 62).

Según el estudio ELAN, como núcleo del diseño de la gestión lingüística, existen factores determinantes relacionados con unos resultados de exportación satisfactorios, que implican el disponer de un personal lingüísticamente competente y/o utilizar traductores-intérpretes profesionales. De hecho, la Guía lingüistica para las empresas europeas (Hagen, 2012), publicada por la Comisión Europea a raíz de este mismo estudio, apunta que si estas invirtieran en los agentes de comunicación multilingüe formando a sus empleados en lenguas o contratando personal profesionalizado en estas, es muy probable que sus exportaciones aumentaran un 50\% más que las de las empresas competidoras que no lo hacen. Lamentablemente, la mayoría de las veces las PYMES españolas prefieren hacer usos puntuales de las empresas de traducción por subcontratación y suponer que su personal de plantilla posee unos conocimientos lingüísticos que, en realidad, suelen ser del todo insuficientes. Esta pobreza profesional impide afrontar las demandas comunicativas de la internacionalización, produciéndose entonces las consecuencias sobre las que avisa el estudio ELAN (Aguayo Arrabal, 2013: 64).

Todo lo expuesto en este capítulo constituye un campo de interés enorme para la Traductología en España, pues genera un flujo de comunicación que engloba una amplia casuística y que se desarrolla a un nivel multicultural y multilingüe (Guillot Farnós, 2015: 2). La adaptación del producto al idioma y cultura locales y el control de los contratos y documentos relevantes que entraña cada remesa de exportación-que, invariablemente, están en inglés como lengua franca de la comunicación mercantil-son factores clave en el éxito de una transacción internacional (Solano Sánchez, 2014). El primer paso para salir al exterior, pues, consiste en pensar en los recursos materiales y humanos que la empresa necesita para afrontar los impedimentos con los que va a tropezar su producto en un mercado ajeno. Entre estos se encuentran su adaptación al mercado meta y los problemas de tipo lingüístico y cultural que dicha adaptación conlleva. Aquí es donde juega el traductor para Comercio Internacional un papel fundamental. 


\section{La formación del traductor como agente de comunicación en el Comercio Internacional. Obstáculos y competencias}

Pese a su potencial idoneidad para ejercer en este campo, existen varios problemas para que el traductor se incorpore inmediata y felizmente a la plantilla de las empresas con vocación internacional.

El primer obstáculo, como ya hemos señalado arriba, proviene de las propias empresas: por razones de confianza o de confidencialidad, o simplemente por economía, el empresario español -hasta tiempos recientes- ha preferido invertir lo mínimo en personal especializado en comunicación, utilizando para estas tareas a los miembros de su plantilla (normalmente dedicados a labores comerciales en el mercado doméstico) procedentes de otras rúbricas profesionales, como Empresariales o Derecho (Mayoral Asensio, 2007: 36; Aguayo Arrabal, 2013: 64), dando por supuesto sus conocimientos lingüísticos. Este es, precisamente, el tipo de comportamiento que reconviene el informe ELAN y que, según este, hace perder dinero a las empresas europeas. De acuerdo con dicho informe, el $75 \%$ de las PYMES españolas no contempla la cualificación lingüística de su plantilla al decidirse a emprender labores de internacionalización, o lo que viene a ser lo mismo, la mayoría de los empleados que se dedican a las tareas lingüísticas de rutina en el marco de la internacionalización no tienen el dominio suficiente del inglés como lengua franca de los negocios, ni de ningún otro idioma. En los peores casos, incluso, como apunta Guillot Farnós (2015: 3), los miembros de la empresa no confían en la labor del traductor en el ámbito del Comercio Internacional como agente capaz de mediar y solucionar los problemas que entraña la comunicación interlingüística. Los problemas que en ella se generan, según Guillot Farnós, se solucionan de forma casi espontánea, confiándose estas labores a no profesionales de la comunicación en situaciones precarias en cuanto a remuneración y plazos.

En segundo lugar, como apunta Mayoral Asensio (2007: 35), hay razones de prurito por parte de los traductores profesionales que trabajan para las empresas, que impiden que estos consideren la actividad de agentes de comunicación en las tareas de Comercio Internacional como propias de su perfil profesional. Estos, al verse obligados a realizar labores administrativas, de gestión de páginas web, de marketing, e incluso del ámbito jurídico, sienten peligrar su identidad profesional. Tal falta de identidad, según creemos, no tiene fundamento alguno. 
Es cierto que las empresas pequeñas y medianas, según añade también Mayoral Asensio (2007: 36), tratan de aprovechar la presencia de un especialista multilingüe para solventar todos los problemas que enfrenta la comunicación internacional con una sola persona. Sin embargo, el traductor internacional debe cobrar consciencia de que, con la necesaria especialización, está del todo capacitado para realizar una larga lista de tareas que entraña la internacionalización, que en nada menoscaban su peso específico profesional. Como señala Aguayo Arrabal (2013: 65):

[....] el traductor en el Comercio Exterior actúa como puente entre el importador y el exportador. Es una pieza clave a la hora de ayudar a establecer relaciones comerciales y a mediar en litigios. Así, sus funciones pueden ir desde la interpretación de enlace en reuniones de negocios a la asistencia a ferias internacionales y otros eventos. Por lo tanto, al estar al frente de la promoción y la asistencia en la empresa, se convierte en el guía y asesor de la relación con los clientes extranjeros.

En tercer lugar, y pese a todo lo expresado anteriormente sobre las competencias reales de que está dotado este tipo de profesional, no está de más que el egresado en Traducción e Interpretación reciba una formación sólida de posgrado o de tipo técnico que lo profesionalice y lo capacite para desarrollar todas las labores que requiere el asesorar al empresario en el proceso de internacionalización. Es cierto que el título de Traductor e Intérprete le capacita para la comprensión, redacción y traducción de textos de Ciencias Sociales (Derecho, Economía y Finanzas, Comercio Internacional) en más de dos idiomas, al tiempo que le dota de destrezas de mediación multilingüe, gestión lingüística, localización y documentación como ninguna otra carrera de lenguas. A estas habilidades hay que añadir, además, las que señala el Libro Blanco que emite la ANECA sobre el título de grado de Traducción e Interpretación, que describen el perfil del traductor como alguien potencialmente preparado para la docencia de lenguas, en competencia con otros títulos como las Filologías. Sin embargo, la profesionalización exige al traductor en el ámbito del Comercio Internacional dar un paso más, pues, tal y como recomienda la Guía básica de la Cámara de Comercio Internacional anteriormente mencionada, una adicional especialización en Comercio Internacional es imprescindible para todo agente que se dedique a este. La profesionalización en este campo afecta al traductor en el ámbito del Comercio Internacional no solo en su capacidad me- 
ramente traductora de documentos de exportación, como bien apunta Socorro Trujillo (2008: 18), sino en una mayor dimensión vocacional, como agente comunicativo en el proceso de internacionalización de las empresas. Para favorecer su plena inserción a este campo hay numerosas instituciones que ofrecen ciclos formativos de Técnico en Comercio Internacional, de acuerdo con lo establecido por el propio Ministerio de Educación y Ciencia (RD 1653/94 BOE 28/09/94 y RD 777/98 BOE 08/05/98). Alternativamente, existen numerosas universidades y escuelas de negocios españolas que ofrecen másteres profesionalizantes en Comercio internacional que pueden funcionar de manera muy sinérgica con un grado de Traducción e Interpretación.

Esta especialización, añadida a las competencias que ya posee el traductor en el ámbito del Comercio Internacional, lo hace idóneo para este campo, ya que le capacita como potencial agente en Comercio Internacional para una serie de requisitos específicos que, según apunta la anteriormente mencionada Guía lingüística de la Comisión Europea (Hagen, 2012: 5), son necesarios para aquellas empresas europeas que deseen internacionalizarse:

- Describir el negocio de forma multilingüe en la página web de la empresa.

- Ayudar al empresario a entender las leyes y normativas locales y a cumplimentar las declaraciones aduaneras.

- Ayudar a formar lingüísticamente a empleados de la empresa para su envío o desplazamiento al extranjero.

- Ayudar al empresario a licitar a concursos públicos y otros tipos de contratos.

- Redactar contratos en el estilo adecuado y de conformidad con las normativas locales.

- Asistir con el empresario o sus empleados a juicios (por ejemplo, para cobrar créditos dudosos y defender patentes) que impliquen la utilización del inglés o de un idioma local.

- Asistir a los especialistas de marketing en la adaptación del producto al mercado local y en el lanzamiento de campañas publicitarias en dicho mercado.

- Asistir lingüística y culturalmente a los especialistas de marketing en la realización de estudios de mercado en un ámbito extranjero. 
- Ayudar al empresario a seleccionar y gestionar un agente o distribuidor local.

- Ayudar al empresario a dirigir y formar trabajadores multilingües a escala nacional y en el extranjero.

- Ayudar al empresario a prestar servicios de asistencia al cliente y garantizar la óptima comunicación en los servicios posventa.

- Asesorar lingüísticamente al empresario o a su representante jurídico para exigir pagos y recuperar créditos dudosos.

- Ayudar al empresario a manejar documentación local, protocolos, estilos internos y especificaciones técnicas.

- Asesorar lingüísticamente al empresario o a su representante jurídico en la negociación de empresas conjuntas, adquisiciones y absorciones.

En resumen, la necesidad de adaptación lingüística de las PYMES españolas a una realidad multilingüística y multicultural hace del papel del traductor en el ámbito del Comercio Internacional una figura clave como facilitador comunicativo, intérprete y mediador.

A continuación nos disponemos a desarrollar más elaboradamente los ámbitos más delicados en los que este profesional puede desarrollar su labor en la empresa que se internacionaliza.

\section{El traductor en el ámbito del Comercio Internacional y su papel en la internacionalización empresarial}

Hemos citado, hasta ahora, las actividades que el traductor puede desarrollar en las empresas, pero no nos hemos adentrado en los aspectos más técnicos que implica esta labor. Solano Sánchez (2014) resume estos aspectos en torno a los siguientes:

1. aspectos materiales y personales,

2. aspectos relacionados con la comercialización del producto,

3. aspectos relacionados con el transporte de la mercancía,

4. aspectos relacionados con la negociación, y

5. aspectos relacionados con la contratación.

En primer lugar, la empresa debe transmitir una imagen corporativa adecuada y profesional, y para ello necesita de un equipo humano sen- 
sible a las cuestiones culturales que son inherentes a la imagen de la empresa y al contacto con los clientes, en el que el traductor como mediador puede jugar un papel fundamental. En este sentido, la página 75 del Libro Blanco del Grado en Traducción e Interpretación describe al traductor como mediador lingüístico y cultural y lo faculta para "la gestión de clientes extranjeros y el trato con interlocutores de otros países (sucursales o filiales, suministradores, autoridades, entidades asociadas, etc.), dentro del trabajo diario en cualquier sector". Asimismo, el traductor en el ámbito del Comercio Internacional puede colaborar a realzar la imagen de la empresa a través de la confección de una página web en inglés y otros idiomas que la describan correctamente, transmitiendo sus valores de forma que estos sean comprensibles lingüística y culturalmente por un público amplio.

Otro ámbito importante es el del Marketing: se han llevado a cabo lanzamientos de productos o servicios que han causado el más estrepitoso de los fracasos, pues la comercialización y distribución del producto están sujetos a delicados matices culturales. La tarea del traductor como mediador en estos procesos es ayudar al mercadólogo a entender qué sucede en la conciencia del comprador entre la llegada de los estímulos del exterior y las decisiones del comprador (Solano Sánchez, 2014: 10). Es esencial, además, que el empresario atienda a un escrutinio profundo de su público objetivo (target market), y al análisis de sus necesidades, reales o creadas. En todos estos ámbitos y en referencia a todos estos factores, constituye una necesidad imperativa el que el traductor-intérprete que se mueve dentro del mundo de la empresa sepa informarse sobre la cultura meta, sabiendo, al tiempo, huir de estereotipos, afirmaciones sin fundamento y lugares comunes sobre esta. Para conocer estos datos y otros, es bueno acudir a las embajadas y cámaras comerciales del país. Además, resulta muy útil familiarizarse con la obra del antropólogo social Edward Hall $(1959,1966)$ y sus estudios sobre proxémica, kinésica y cronémica, así como con los más recientes estudios de los psicólogos y sociólogos especializados en cultura empresarial Mole (1998), Trompenaars (1997), Klopf (1991), y Hofstede (2010).

En tercer lugar, el Comercio Internacional está apoyado sobre usos y sistemas documentarios que traducen los derechos, costes y responsabilidades del proceso mismo a los documentos que los expresan, ya que la actividad internacional tiene dos facetas: el envío real de la mercancía y 
el intercambio de los documentos que a esta acompañan. Para desempeñar su labor de forma rigurosa, el traductor en el ámbito del Comercio Internacional debe conocer los códigos internacionales que representan dónde cambia la posesión y el riesgo de la mercancía entre exportador e importador (INCOTERMS) y los documentos que acompañan a dicha mercancía, ya que en el proceso de exportación reviste tanta importancia proporcionar los documentos correctos como suministrar la mercancía correcta. Según Socorro Trujillo (2008) y Bertasi (2012: 15) estos documentos clave son:

1. el contrato de compraventa,

2. el conocimiento de embarque (u otro documento de transporte),

3. los documentos relativos al pago (especialmente el crédito documentario y la letra de cambio o el efecto bancario), y

4. el documento de seguro (póliza, certificado o nota de cobertura).

Así, el traductor que se dedica a la exportación-importación debe conocer estos documentos internacionales de transporte, seguro y pago con cierta profundidad y debe saber tratarlos con respeto, con el fin de aconsejar a los demás participantes en el proceso de internacionalización de la empresa si se generase ambigüedad de significado en un caso dado. Al fin y al cabo, uno de los riesgos del sistema documentario es que los documentos en papel son susceptibles de ser falsificados, alterados o sencillamente interpretados de forma errónea (Bertasi, 2012: 16). De hecho, y pese a que las que estos documentos contienen son, a menudo, cláusulas paradigmáticas (boiler-plate) y están mayoritariamente en inglés como lengua franca de la profesión, la dificultad que dichas cláusulas poseen reside, no en su significado literal, sino en su correcta interpretación (Mayoral Asensio, 2007). Un conocimiento profundo de estas desde el punto de vista lingüístico y pragmático por parte un especialista en lenguas profesionales como es el traductor implica una preparación preliminar sobre los fines para los que están concebidas, el contexto en el que se utilizan y el papel que esas cláusulas juegan en las operaciones de exportación-importación.

Por otro lado, el negociar es siempre un proceso complejo incluso en el ámbito doméstico, pero negociar entre culturas es todavía más complicado, debido a las posibilidades de malos entendidos que nacen de las diferencias culturales, pues los estilos de negociación difieren de país a país. Según López y Roca (2005), los españoles somos afortuna- 
dos, porque nuestro estilo de negociación es lo suficientemente híbrido desde el punto de vista comunicativo como para que nos adaptemos fácilmente a distintos contextos comerciales. Sin embargo, según estos autores, los empresarios españoles carecen, por lo general, de dominio de idiomas y no suelen tener paciencia con los intérpretes, utilizando interrupciones, tonos agresivos y excesivas gesticulaciones. De este modo, sus oponentes extranjeros se constituyen en receptores indirectos de un exceso verbal y gestual, observando a menudo perplejo cómo el español se dirige únicamente al traductor-intérprete, como discutiendo con él. Es aquí donde debe actuar de nuevo este profesional como mediador cultural, persuadiendo al cliente u oponente no nativo de que la diatriba no es amenazante, ni está dirigida a sí mismo como intérprete del intercambio, sino que es parte del nudo de la negociación. Según varios autores, en el proceso de mediación cultural que tiene en lugar en el marco de la empresa que se internacionaliza, en la labor del traductor (Taft, 1981; Hatim y Mason, 1990) y en la del intérprete (Kondo, 1990; Brislin, 1981; Werner, Knapp-Potthof y Knapp, 1987) es fundamental el empleo de su capacidad de comunicador, reconociendo el condicionamiento al que le somete su propia cultura (cultural self-awareness) y la cultura o culturas meta.

Finalmente, en el Comercio Internacional los acuerdos contractuales pueden ser mucho más susceptibles a complicaciones que los acuerdos domésticos. Las barreras idiomáticas pueden causar malentendidos y pueden surgir obstáculos culturales y geográficos; incluso el significado de algunas palabras puede diferir de un lugar a otro. De este modo, los contratos comerciales internacionales deben ser específicos y abarcar todos los aspectos de la transacción, reduciéndose así el riesgo de ambigüedades, malinterpretaciones y disputas (O’Connor, 2012: 30). En este punto, cabe afirmar que el traductor debe tener un conocimiento general de los principios jurídicos que se aplican a las operaciones internacionales y conocer los sistemas jurídicos que están involucrados en las leyes aplicables al contrato (básicamente, el Common Law anglosajón y el Derecho Continental europeo, así como, en algunos casos, la ley islámica conocida como ley Shari'a). Como recuerda la Guía lingüística para las empresas europeas (Hagen, 2012: 26), un simple proceso comunicativo como es la redacción e interpretación de contratos de compraventa en el ámbito internacional nunca es, simplemente, un proceso de traducción. 


\section{El traductor en el ámbito del Comercio Internacional desde el prisma de la Traductología: traducir a través de géneros}

Para terminar este trabajo nos gustaría hacer un pequeño apunte sobre lo que significa la labor del traductor en el ámbito del Comercio Internacional desde la perspectiva de la Traducción como disciplina de trabajo e investigación. Como hemos visto en nuestros anteriores capítulos, el traductor en el ámbito del Comercio Internacional no deja de ser un tipo muy particular de traductor especializado, poseyendo conocimientos que han de ser, necesariamente, de naturaleza económica, jurídica y empresarial. Sin embargo, y pese disponer de todas las competencias que hemos descrito como esenciales para el desarrollo de su labor, el traductor de textos internacionales no tiene por qué ser un especialista, practicante del Marketing, el Derecho o la Economía, para conocer muy bien los resortes que activan la comunicación en la comunidad profesionalizada en la que se mueve. Como apunta Gamero (2001: 42), para traducir textos especializados de forma rigurosa y efectiva resulta imperativo conocer el campo temático, la terminología especializada y los recursos documentales de la disciplina hacia y desde la que se traduce, además de -y de forma esencial- haber adquirido una competencia genérica sobre sus textos: lo que se ha dado en llamar genre literacy (Spack, 1988:36).Dicho de otro modo, el traductor especializado en este ámbito no se constituye de forma estricta como un especialista en Comercio Internacional, conociendo y operando en la disciplina o disciplinas en las que se mueve de forma tan exhaustiva como dicho especialista. Empero, sí puede -y debe- estar familiarizado con los géneros que de estas emanan, con el fin de adentrarse en los propósitos comunicativos y en los mecanismos operativos de esas disciplinas.

En este punto, cabría la necesidad de definir lo que aquí se concibe como género y la importancia que este constructo tiene en la Traducción Especializada. Un género, o sistema de géneros, no es sino la herramienta comunicativa esencial por medio de la cual operan y evolucionan las comunidades profesionales. Por lo tanto, cuanto más conocimiento genérico tenga el traductor especializado de la profesión a la que traduce, mayor conocimiento "iniciático" tendrá sobre los mecanismos que articulan su comunicación (Orts, en prensa). En este sentido, cabe apuntar que no nos referimos a un mero conocimiento superficial de la tipología textual del Comercio Internacional y de sus rasgos 
formales o lingüísticos, que correspondería a un sistema artificial de clasificación de textos (Tessutto, 2012). Esta noción cabría más en una conceptualización funcionalista del texto a nivel de registro (Christie y Martin, 1997, por ejemplo, en Luzón, 2005: 135), estudios que ya han quedado superados al haber sido integrados, de forma evolutiva dentro de la Traductología, en los estudios de Traducción Especializada como actividad enmarcada necesariamente en la utilización de géneros. Al fin y al cabo, el registro no es sino la forma en que se materializa lingüísticamente el género como acto comunicativo $\mathrm{y}$, como hemos visto, el trabajo del traductor de textos internacionales proyecta su labor más allá de lo puramente lingüístico, constituyéndose como mediador en los procesos comunicativos que articulan las transacciones de exportación e importación.

Para aclarar la diferencia entre una visión plana y una integral de lo que constituye la traducción especializada como proceso de mediación social, se hace necesario poner de relieve la manera de contemplar esta disciplina por parte de Hatim y Mason (1990: 105), García Izquierdo (2000) y Schäffner (2002: 210-212), entre otros, quienes distinguen entre tipo de texto, o modelo clasificatorio basado en el tema o ámbito disciplinario, y género textual, concebido desde su total dimensión social y comunicativa. Siguiendo esta vertiente que contempla el género como vital para el proceso traductológico, Gamero (2001: 38) diferencia entre el control por parte del traductor de los mecanismos "intratextuales" y de los «extratextuales» que operan en las fases de traducción de un texto especializado. Esta división de Gamero evidencia que el traductor que se mueve en el ámbito de las disciplinas profesionales debe ser consciente tanto de los factores léxico-gramaticales a nivel de registro y de su uso restrictivo en cada género (dimensión intratextual), como de los mecanismos retórico-discursivos que se asocian a dicho género, y que forjan el propósito comunicativo de este, otorgándole, de esta manera, una vertiente pragmática (dimensión extratextual). Dicho control no es sino el elemento que va a tornar al traductor de textos internacionales en un mediador en los procesos de comunicación que tienen lugar en las transacciones de exportación e importación, aportándole la dimensión social que necesita para que los propios especialistas en la disciplina lo reconozcan como eslabón ineludible en la actividad comunicativa del Comercio Internacional. 
De forma adicional, cabe añadir que, en concreto, la comunidad profesional que constituye el Comercio Internacional no emite un solo tipo de géneros, sino que está organizado alrededor de lo que se ha dado en llamar una "colonia de géneros" (Bhatia 2004: 59, en Pizarro, 2010:13), o conjunto de modelos textuales flexibles que se adecúan a las necesidades sociocognitivas de las comunidades discursivas de las que parten (Luzón, 2005: 135), en este caso, la comunidad discursiva del Comercio Internacional. Este concepto de colonia de géneros como haz comunicativo de una disciplina tiene mucho sentido en el ámbito de la actividad comercial de exportación-importación, pues, como hemos visto en nuestro anterior apartado, la labor de internacionalización de productos es, en muchos de sus aspectos, una labor apoyada en las remesas documentarias, en las que dichos productos van acompañados -desde su origen en el país de exportación hasta su destino en el de importación- de diferentes fases documentales. Primeramente, tendría lugar una fase puramente comercial o de compraventa en la que predomina el manejo de una variada gama de géneros contractuales (contratos de compraventa, cláusulas de exportación o Incoterms, por ejemplo), pasando por una fase logística en la que son esenciales los géneros que mueven el ámbito del transporte y los seguros (conocimientos de embarque, pólizas del Instituto de Aseguradores de Londres, entre otros muchos). Finalmente, y como culmen de cualquier transacción de compraventa en el comercio internacional, estaría la fase del pago, donde son instrumentales todos los géneros que se encaminan a este propósito y en la que se encontrarían, fundamentalmente, el propio crédito documentario -como medio de pago más habitual en las transacciones internacionales-, pero también otros modos más simples como la letra de cambio o el efecto bancario.

Es el conocimiento y manejo de todos estos documentos, no como una simple tipología textual que se repite en un proceso estandarizado, sino como géneros, o productos de la actividad comunicativa en virtud la cual se articulan estas complejas transacciones, lo que va a convertir al traductor de textos internacionales en un nexo comunicativo fundamental, con una marcada labor social, reconocida por la disciplina profesional que tiene como propósito y función la internacionalización del comercio. 


\section{Conclusión}

Efectivamente, España es un país que se vuelca al exterior con la apertura de sus mercados a la internacionalización y, consecuentemente, al contacto con diferentes países y sus lenguas y culturas. Por ello, uno de los elementos de más relevancia en esa internacionalización es la adopción de estrategias lingüísticas que hagan frente a los problemas de comunicación en los mercados nuevos o en expansión de los países a los que se dirige. Según lo expuesto a lo largo de este trabajo, no hay ninguno de los aspectos relacionados con esta cuestión que no pueda ser afrontado de manera exitosa por un traductor-intérprete que esté formado en Comercio Internacional, y que ese halle preparado para actuar como agente e intermediario comunicativo clave en el proceso. Al fin y al cabo, su papel es esencial en todos esos aspectos, pero fundamentalmente en la esencia de lo que constituye un proceso exportador: la redacción e interpretación, así como el control, de los documentos esenciales que forman las remesas documentarias que entraña una transacción de este tipo.

En definitiva, cuando el traductor en el ámbito del Comercio Internacional se constituye en un agente más de la internacionalización y conoce de todos sus ámbitos (negociación, contratación, logística, comercialización), cobra consciencia de su propio papel, del papel que deben jugar los distintos participantes en él y de cuál puede ser su labor para optimizar todo el proceso de salida de su empresa al mercado internacional.

\section{Referencias bibliográficas}

Aguayo Arrabal, Natividad. 2013. El traductor-intérprete en el Comercio Exterior: ¿Realidad o necesidad? Entreculturas 5: 57-74.

Bertasi, Stephano. 2012. Preámbulo: introducción a la práctica del comercio internacional. En Cámara de Comercio Internacional. 2013. Guía básica del comercio internacional. Amazon, edición Kindle, 10-17.

Brislin, Richard W. 1981. Cross cultural encounters, face-to-face interaction. California: Pergamon Press.

Cámara de Comercio Internacional. 2013. Guía básica del comercio internacional. Amazon, edición Kindle.

Comisión Europea. 2006. Informe ELAN, Effects on the European Economy of Shortages of Foreign Language Skills in Enterprise. http://ec.europa. 
eu/languages/policy/strategic-framework/documents/elan_en.pdf [Acceso 25/10/2016].

Gamero, Silvia. 2001. La traducción de textos técnicos: descripción y análisis de textos (alemán-español). Barcelona: Ariel.

García Izquierdo, Isabel. 2000. Análisis textual aplicado a la traducción. Valencia: Tirant Lo Blanch.

Guillot Farnós, Antonio. 2015. La traducción en el transporte marítimo. Descripción de un campo profesional y sus políticas de traducción. [Tesis Doctoral inédita]. Castellón: Universidad Jaume I, Departamento de Traducción y Comunicación.

Instituto Español de Comercio Exterior. 2015. Invest in Spain. Invertir y hacer negocios en España. Madrid: ICEX. http://www.investinspain.org/invest/es/index.html [Acceso 25/10/2016].

Hagen, Stephen. 2011. La Guía lingüistica para las empresas europeas. Comisión Europea. Luxemburgo: Oficina de Publicaciones de la Unión Europea. http://bookshop.europa.eu/es/la-gu-a-lingue-stica-para-lasempresas-europeas-pbNC3110923/ [Acceso 25/10/2016].

Hatim, Basil \& Mason, Ian. 1990. Discourse and the Translator. Language in Social Life Series. Londres/Nueva York: Longman.

Hall, Edward T. 1959. The Silent Language. New York: Doubleday.

Hall, Edward T. 1966. The Hidden Dimension. New York: Doubleday.

Hofstede Geert. 2010. Cultures and Organisations, Software of the Mind. Nueva York: McGraw-Hill.

Katan, David. 1999. Translating Cultures: An Introduction for Translators, Interpreters and Mediators. Manchester: St Jerome.

Klopf, Donald. 1987. Workbook for Intercultural Encounters: The fundamentals of intercultural communications. Englewood, CO: Morton Publishing Company.

Kondo, Masaomi. 1990. What conference interpreters should not be expected to do. The Interpreter's Newsletter 3: 59-65.

LópezMartínez, Félix \& Roca Aymar, José Luís. 2005. Negociación y Contratación. En Curso Superior. Estrategia y Gestión del Comercio Exterior. ICEX y Consejo Superior de Cámaras de Comercio, Industria y Navegación de España (CSC).

Luzón, María José. 2005. Aplicación del concepto de "colonia degéneros" a la enseñanza de Inglés para Fines Específicos. Ibérica 10: 33-144.

Mayoral Asensio, Roberto. 2007. La traducción comercial. En Fuertes Olivera, Pedro A. Problemas lingüísticos en la traducción especializada. Valladolid: Universidad de Valladolid, Secretariado de Publicaciones e Intercambio Editorial, 33-48. 
Mole, John. 1998. Mind your Manners. Managing Business Cultures in Europe. Londres: Nicholas Brealey Publishing.

O’Connor, Emily. 2012. El marco jurídico de la exportación y la importación. En Cámara de Comercio Internacional. Guía básica del comercio internacional. Amazon, edición Kindle, 25-33.

Orts, María Ángeles. En prensa. El género como método de estudio y enseñanza en la traducción de textos especializados en inglés-español; el género y la traducción jurídica. En Rojo, Ana (ed.) La investigación en traducción: Aspectos metodológicos y aplicaciones didácticas. Barcelona: Anthropos.

Pizarro, Isabel. 2010. Análisis y traducción del texto económico inglés-español. La Coruña: Netbiblo.

Schäffner, Christina (ed.). 2002. The Role of Discourse Analysis for Translation and in Translator Training. Clevedon/Buffalo/Toronto/Sydney: Multilingual Matters Ltd.

Socorro Trujillo, Karina. 2008. Aspectos textuales y terminológicos de documentos mercantiles del comercio internacional. Las Palmas: Servicio de Publicaciones de la Universidad de Las Palmas de Gran Canaria.

Solano Sánchez, Miguel Ángel. 2013. Exportar es fácil, si sabes cómo. Guía rápida de exportación. Amazon, edición Kindle.

Spack, Ruth. 1988. Initiating ESL students into the academic discourse community: How far should we go? TESOL Quarterly 22(1): 29-51.

Taft, Ronald. 1981. The role and personality of the mediator. En Bochner, Stephen. The Mediating Person: Bridges Between Cultures. Boston: Schenkman Publishing Co., 53-88.

Tessuto, Girolamo. 2012. Investigating English Legal Genres in Academic and Professional Contexts. Cambridge: Cambridge Scholars Publishing.

Trompenaars, Fons \& Hampden-Turner, Charles. 1998. Riding the Waves of Culture. Londres: Nicholas Brealey Publishing.

Werner, Enninger; Knapp, Karlfried \& Knapp-Potthoff, Annelie. 1987. Analyzing Intercultural Communication. Berlin: Mouton de Gruyter. 
